\title{
Biochemical and clinical benefits of unilateral adrenalectomy in patients with subclinical hypercortisolism and bilateral adrenal incidentalomas
}

\author{
I Perogamvros, D A Vassiliadi ' , O Karapanou, E Botoula, M Tzanela and S Tsagarakis \\ Department of Endocrinology, Diabetes and Metabolism, Evangelismos Hospital, 10676 Athens, Greece and \\ ${ }^{2}$ Endocrine Unit, Second Department of Internal Medicine-Propaedeutic, Research Institute and Diabetes Center, \\ Attiko University Hospital, 12462 Athens, Greece
}

Correspondence should be addressed to $S$ Tsagarakis Email stsagara@otenet.gr

\begin{abstract}
Objective: The treatment of subclinical hypercortisolism in patients with bilateral adrenal incidentalomas (Al) is debatable. We aimed to compare the biochemical and clinical outcome of unilateral adrenalectomy vs a conservative approach in these patients.

Design: Retrospective study.

Methods: The study included 33 patients with bilateral Al; 14 patients underwent unilateral adrenalectomy of the largest lesion (surgical group), whereas 19 patients were followed up (follow-up group). At baseline and at each follow-up visit, we measured $0800 \mathrm{~h}$ plasma ACTH, midnight serum cortisol (MSF), 24-h urinary-free cortisol (UFC) and serum cortisol following a standard 2-day low-dose-dexamethasone-suppression test (LDDST). We evaluated the following comorbidities: arterial hypertension, impaired glucose tolerance or diabetes mellitus, dyslipidemia and osteoporosis.

Results: Baseline demographic, clinical characteristics and the duration of follow-up $(53.9 \pm 21.3$ vs $51.8 \pm 20.1$ months, for the surgical vs the follow-up group) were similar between groups. At the last follow-up visit the surgical group had a significant reduction in post-LDDST cortisol ( $2.4 \pm 1.6 \mathrm{vs} 6.7 \pm 3.9 \mu \mathrm{g} / \mathrm{dl}, P=0.002)$, MSF $(4.3 \pm 2 \mathrm{vs} 8.8 \pm 4.6 \mu \mathrm{g} / \mathrm{dl}, P=0.006)$ and $24-\mathrm{h}$ UFC $(50.1 \pm 21.1 \mathrm{vs} 117.9 \pm 42.4 \mu \mathrm{g} / 24 \mathrm{~h}, P=0.0007)$ and a significant rise in mean \pm s.D. morning plasma ACTH levels $(22.2 \pm 9.6 \mathrm{vs}$ $6.9 \pm 4.8 \mathrm{pg} / \mathrm{ml}, P=0.002$ ). Improvement in co-morbidities was seen only in the surgical group, whereas no changes were noted in the follow-up group.

Conclusions: Our early results show that removal of the largest lesion offers significant improvement both to cortisol excess and its metabolic consequences, without the debilitating effects of bilateral adrenalectomy. A larger number of patients, as well as a longer follow-up, are required before drawing solid conclusions.
\end{abstract}

\section{Introduction}

Incidentally detected adrenal masses, known as adrenal incidentalomas (AI), have become a very common clinical problem, with an estimated prevalence that ranges between 0.4 and $7 \%$ and increases with age (1). In terms of hormone hypersecretion, hypercortisolism is the most commonly observed disorder, with an incidence of $1-47 \%$, depending on the adopted diagnostic criteria (2).
In the setting of AI, hypercortisolism is usually not manifested by the clinical stigmata of cortisol excess, and therefore it is described as 'mild' or 'subclinical' Cushing's syndrome or, more frequently, as subclinical hypercortisolism ( $\mathrm{SH})$. Although the clinical implications of subclinical hypercortisolism remain elusive, various abnormalities, such as diabetes, hypertension, obesity, (c) 2015 European Society of Endocrinology Printed in Great Britain
Published by Bioscientifica Ltd 
dyslipidemia and osteoporosis, are frequently observed in these patients $(3,4)$.

Contrary to the diagnosis and management of overt adrenal Cushing's syndrome that is usually straightforward, there is currently no consensus on both the diagnosis and the treatment of subclinical hypercortisolism associated with AI. The diagnostic criteria vary widely between centres $(2,5)$. Treatment is also controversial. For unilateral $\mathrm{AI}$ associated with $\mathrm{SH}$, limited data suggest that surgical treatment may result in some beneficial metabolic effects $(5,6,7)$. There is, however, insufficient evidence to routinely support adrenalectomy and, currently, a patient-based approach is advocated. About 9-17\% of AI are bilateral and, as previously shown by our group, these patients have a higher prevalence of $\mathrm{SH}$ compared to their unilateral counterparts (8). If surgery, however, is a dilemma for patients with unilateral adenomas, it is an even more complex decision for patients with bilateral AIs. Bilateral adrenalectomy will necessitate lifelong adrenocortical replacement, which practically converts mild endogenous hypercortisolism to mild exogenous hypercortisolism, as many of these patients are overreplaced (9). Alternate options include a conservative approach with rigorous management of co-morbidities or excision of the largest lesion, based on observations that the size of the adrenal lesion correlates with the degree of cortisol excess. Unilateral adrenalectomy therefore has been suggested as an attractive option due to its lower complications, but the evidence on its efficacy is scarce $(10,11,12)$. In this study we aimed to compare the biochemical and clinical long-term outcome of unilateral adrenalectomy vs a conservative approach in patients with bilateral AIs.

\section{Subjects and methods}

We studied 33 patients (28 females) with bilateral AIs of more than $1.5 \mathrm{~cm}$ diameter who presented to the Department of Endocrinology, Diabetes and Metabolism, at Evangelismos Hospital in the last decade (2003-2013). All adrenal masses were discovered by ultrasound or computerized tomography (CT) scan of the abdomen performed for reasons unrelated to an adrenal disorder. All AI originally discovered by ultrasound were confirmed by CT scanning. They all had features suggestive of benign adrenocortical adenomas (homogeneous, smoothly marginated lesions). A Hounsfield unit cut-off of $<10$ was used to indicate a benign imaging phenotype. In patients with higher values, lesions with an absolute and/or relative contrast washout value of more than $60 \%$ or a loss of signal intensity of $20 \%$ or greater on out-of-phase MRIs were also considered benign. All but five patients had one nodule on each side. Four patients had two adenomas on one side and one adenoma on the other side, whereas in one patient, both adrenal glands were enlarged with multiple macronodules. Based on pathology reports, histologically eight patients had only one adenoma, with or without hyperplasia of the adjacent adrenal tissue, whereas six patients had a dominant nodule and multiple micro- and macronodules and one patient had diffuse hyperplasia.

Patients had no specific symptoms or signs of overt cortisol hypersecretion (i.e., proximal myopathy, easy bruising, striae, facial plethora). Phaeochromocytomas and aldosteronomas were excluded by appropriate hormonal testing (24-h urinary concentrations of VMA, metanephrines, normetanephrines and screening with aldosterone/plasma renin concentration, respectively). A number of patients were tested for aberrant receptors following a modified protocol (13); six in in the intervention group and nine in the follow-up group. Among those tested, two tested positive, two had borderline responses to posture test in the surgery group and two tested positive (one in posture test, the other in meal test) in the follow-up group.

Based on informed patient preference, 14 patients (all women, age $54.9 \pm 6.7$ years) had unilateral adrenalectomy (group A) and 19 patients (14 women, age 59.0 \pm 8.7 years) underwent observation and medical treatment of comorbidities (group B). Group A patients underwent laparoscopic resection of the adrenal with the largest adenoma or the left adrenal in case the masses at both sides were of equal size.

All patients were investigated as inpatients in a controlled clinical environment, under unstressed conditions. The following parameters were measured at each routine follow-up visit, but only the baseline and final follow-up visit values are reported: $0800 \mathrm{~h}$ plasma adrenocorticotrophic hormone (ACTH), midnight serum cortisol (MSF), serum cortisol following a standard 2-day low-dose dexamethasone suppression test (LDDST, $0.5 \mathrm{mg}$ every $6 \mathrm{~h}$ for 2 days) and a 24-h urinary-free cortisol (UFC). SH was diagnosed based on previously published diagnostic criteria (8), post-LDDST cortisol levels $>1.8 \mu \mathrm{g} / \mathrm{dl}$ and at least one of the following: ACTH levels $<10 \mathrm{pg} / \mathrm{ml}$, MSF $>7 \mu \mathrm{g} / \mathrm{dl}$ and UFC above the normal range for our assay ( $>120 \mu \mathrm{g} / 24 \mathrm{~h})$. Patients with ACTH more than $10 \mathrm{pg} / \mathrm{ml}$ were investigated for the possibility of Cushing's disease by a pituitary MRI scan and/or desmopressin (DDAVP) test. None of the patients 
tested positive and none of the patients developed evidence of Cushing's disease during follow-up.

The presence of comorbidities associated with AIs and SH was evaluated at baseline and at each follow-up visit. At each follow-up evaluation we recorded the following parameters: current medications, in-hospital and selfrecorded blood pressure measurements, glucose selfmonitoring (in those with known diabetes) and any cardiovascular-related events. We also measured lipid levels as well as HbAlc and/or performed an oral glucose tolerance test (OGTT). Arterial hypertension (AH) was defined as systolic blood pressure $\geq 140 \mathrm{mmHg}$ and/or diastolic blood pressure $\geq 90 \mathrm{mmHg}$ and/or the administration of antihypertensive medication. Diabetes mellitus (DM) and impaired glucose tolerance (IGT) were diagnosed based on established criteria (14). Dyslipidemia was characterized based on serum total cholesterol levels $>200 \mathrm{mg} / \mathrm{dl}$, in combination with LDL cholesterol $>170 \mathrm{mg} / \mathrm{dl}$ or HDL cholesterol $<40 \mathrm{mg} / \mathrm{dl}$ in men and $<50 \mathrm{mg} / \mathrm{dl}$ in women and/or fasting serum triglyceride levels $>150 \mathrm{mg} / \mathrm{dl}$ and/or the administration of lipidlowering medication. We assessed bone mineral density with Dual Energy X-ray Absorptiometry (DEXA) and diagnosed osteoporosis when the T-score at the measured site was -2.5 or less.

We defined as improvement of the co-morbidities under investigation the improvement of biochemical markers if no medication was used and the use of decreased dosages or discontinuation of relevant medication. Improvement of osteoporosis was defined as a T-score of $>-2.5$ during follow-up.

\section{Laboratory assays}

We measured cortisol with a chemiluminescence immunoassay (CLIA) using the ADVIA Centaur Immunoassay System. We measured UFC after dichloromethane extraction with the same CLIA. ACTH was measured by an immunoradiometric assay (Cis bio international, Gif sur Yvette, France).

\section{Statistical analysis}

Statistical analysis was undertaken using Graphpad Prism version 5.0 (Graphpad Software, Inc., San Diego, CA, USA). Data are reported as mean \pm s.D. Biochemical parameters were compared using the Mann-Whitney non-parametric test or the Wilcoxon matched pairs test, where appropriate. The $\chi^{2}$-test was used to compare the proportions of patients with improvement in co-morbidities between the two groups. $P \leq 0.05$ was considered statistically significant.

\section{Results}

Baseline demographic and clinical characteristics were not statistically different between groups A and B (Table 1). These included sex, age at diagnosis, BMI, waist-to-hip ratio (WHR), size of largest adenoma, duration of follow-up and incidence of subclinical hypercortisolism, hypertension, disorders of carbohydrate metabolism, dyslipidemia and osteoporosis.

In group A, no major surgical complications were reported. Post-operatively, seven of 14 patients were put on hydrocortisone (for up to 6 months) on the basis of either low morning cortisol levels or clinical symptoms of adrenal insufficiency.

Post-surgery, we noted a significant decrease (Fig. 1, last follow-up visit vs baseline values reported) in mean \pm s.D. levels of serum cortisol levels following the LDDST $(2.4 \pm 1.6$ vs $6.7 \pm 3.9 \mu \mathrm{g} / \mathrm{dl}, P=0.002)$, MSF $(4.3 \pm 2$ vs $8.8 \pm 4.6 \mu \mathrm{g} / \mathrm{dl}, P=0.006)$ and 24 -h UFC $(50.1 \pm 21.1$ vs $117.9 \pm 42.4 \mu \mathrm{g} / 24 \mathrm{~h}, P=0.0007)$, and a significant rise in mean \pm s.D. morning plasma ACTH levels $(22.2 \pm 9.6$ vs $6.9 \pm 4.8 \mathrm{pg} / \mathrm{ml}, P=0.002$ ).

This improvement in biochemical parameters in group A was obvious in the majority of patients when

Table 1 Demographic and clinical characteristics and improvement in the co-morbidities at last follow-up in group $A$ (unilateral adrenalectomy) and group B (simple observation). Size refers to the size of the largest adenoma. Continuous variables are presented as mean \pm s.D.

\begin{tabular}{|c|c|c|c|}
\hline Characteristics & $\begin{array}{c}\text { Group A } \\
(n=14)\end{array}$ & $\begin{array}{c}\text { Group B } \\
(n=19)\end{array}$ & $\boldsymbol{P}$ \\
\hline Sex (F/M) & $14 / 0$ & $14 / 5$ & NS \\
\hline Age & $54.9 \pm 6.7$ & $59 \pm 8.7$ & NS \\
\hline BMI & $27.3 \pm 7.3$ & $28 \pm 4.6$ & NS \\
\hline WHR & $0.9 \pm 0.1$ & $0.92 \pm 0.1$ & NS \\
\hline Size $(\mathrm{cm})$ & $4.1 \pm 0.6$ & $3.7 \pm 0.8$ & NS \\
\hline Follow-up (months) & $53.9 \pm 21.3$ & $51.8 \pm 20.1$ & NS \\
\hline $\mathrm{SH}$ & $12 / 14$ & $11 / 19$ & NS \\
\hline HT & 7 & 12 & NS \\
\hline Improvement in HT & $4 / 7$ & $0 / 12$ & 0.009 \\
\hline DM2 and IGT & 4 & 4 & NS \\
\hline Improvement in DM2 or IGT & $4 / 4$ & $0 / 4$ & 0.02 \\
\hline Dyslipidemia & 8 & 6 & NS \\
\hline Improvement in dyslipidemia & $2 / 8$ & $0 / 6$ & NS \\
\hline Osteoporosis & 3 & 5 & NS \\
\hline Improvement in osteoporosis & $2 / 3$ & $0 / 5$ & 0.03 \\
\hline
\end{tabular}

NS, not statistically significant 

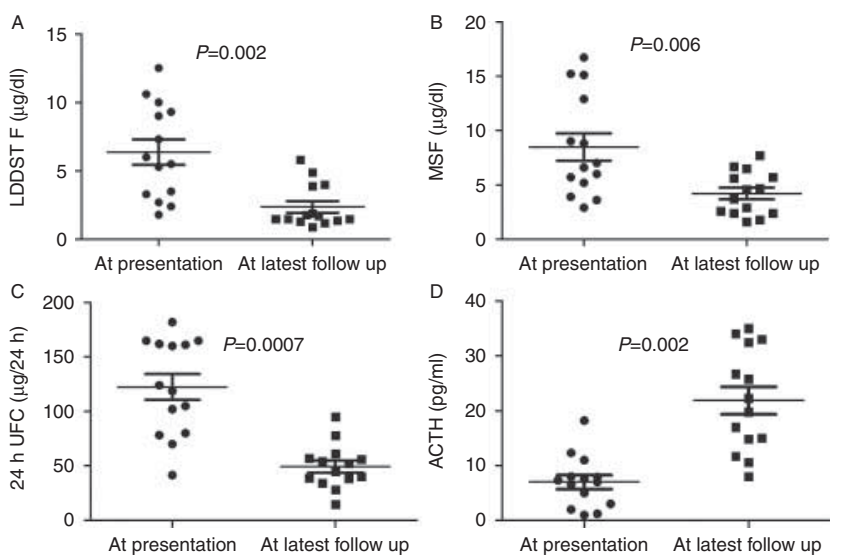

\section{Figure 1}

Biochemical parameters before and after unilateral adrenalectomy in group A. (A) LDDST F, serum cortisol following a standard 2-day low-dose dexamethasone suppression test; (B) MSF, midnight serum cortisol; (C) 24-h UFC, 24-h urinary free cortisol; (D) $0800 \mathrm{~h}$ plasma ACTH.

normal cut-offs were considered. At the end of the followup period (vs baseline), ten of 14 (vs 0 of 14) patients had normal post-LDDST serum cortisol, 13 of 14 (vs seven of 14) patients had normal MSF, all patients (vs seven of 14) had normal 24-h UFC and 13 of 14 (vs three of 14) had normal plasma ACTH. Accordingly, the incidence of $\mathrm{SH}$ fell dramatically post-surgery (one of 14) compared to baseline (12 of 14). In contrast, hormonal characteristics remained unchanged in group B (Fig. 2).

Improvement and/or normalization of co-morbidities were achieved in group A but not in group B (Tables 1 and 2). More specifically, blood pressure was normalized off medications or antihypertensive medications were discontinued post-adrenalectomy in four of seven patients with $\mathrm{AH}$, a normalization was noted in all four patients with disorders of carbohydrate metabolism (DM2 or IGT), two of eight discontinued their lipid lowering drugs and two of three patients with osteoporosis had improved bone mineral density scores post-surgery (T-score <2.5). In comparison, there was no improvement in any of the co-morbidities in the group B patients. This difference between groups reached statistical significance for hypertension (HT), DM2, IGT and osteoporosis but not dyslipidemia (Table 1). A number of patients in both groups did not fulfil the criteria for $\mathrm{SH}$, although most had at least one biochemical abnormality. When we analysed separately the SH-only subjects in both groups, the improvement in comorbidities remained significant (Table 3).

\section{Discussion}

This is the first study to show that unilateral adrenalectomy leads to an improvement in biochemical and clinical aspects of subtle cortisol hypersecretion in patients with bilateral adrenal adenomas, with clear advantages over simple observation. The adrenal gland with the largest adenoma was preferentially removed laparoscopically, or the left adrenal in the case of bilateral adenomas of the same size, due to the less demanding nature of this approach. In our cohort, no major complications resulted from surgery. Importantly, improvement in biochemical parameters of cortisol secretion was observed in all patients, with complete normalization in most. This was clinically reflected by improvement in hypertension, hyperglycemia and osteoporosis, No clinical or biochemical improvement was noted over time in patients who did not undergo surgery.

Although bilateral adrenalectomy is generally considered the treatment of choice for overt Cushing's syndrome secondary to bilateral adrenal hyperplasia (primary pigmented nodular adrenal dysplasia (PPNAD) or ACTH-independent macronodular adrenal hyperplasia (AIMAH)), it remains debatable whether it is advantageous in patients with bilateral AIs without overt Cushing's syndrome. This is because, on the one hand, the clinical situation is not severe enough to prompt surgical management, and on the other hand, the patient is lifelong dependent on steroid replacement and at risk for
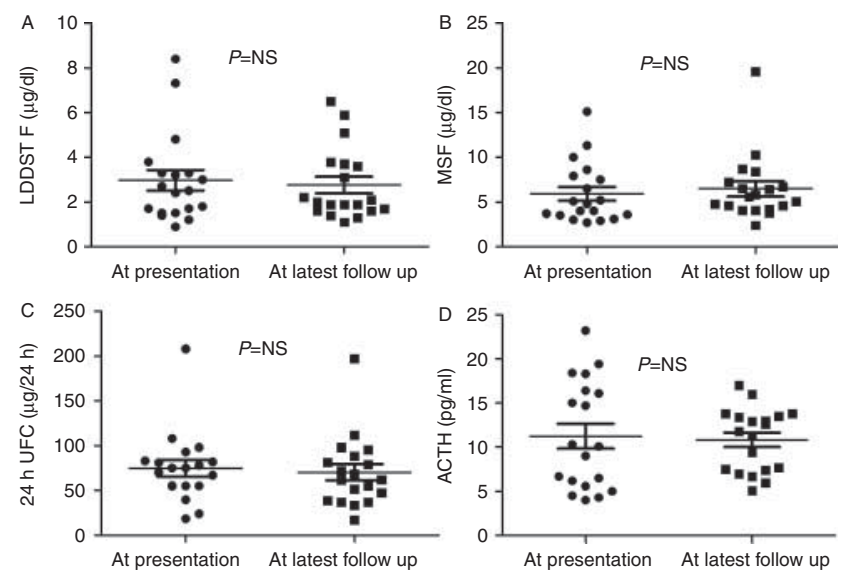

\section{Figure 2}

Biochemical parameters at baseline and last follow-up visit in group B. (A) LDDST F, serum cortisol following a standard 2-day low-dose dexamethasone suppression test; (B) MSF, midnight serum cortisol; (C) 24-h UFC, 24-h urinary free cortisol;

(D) $0800 \mathrm{~h}$ plasma ACTH. 


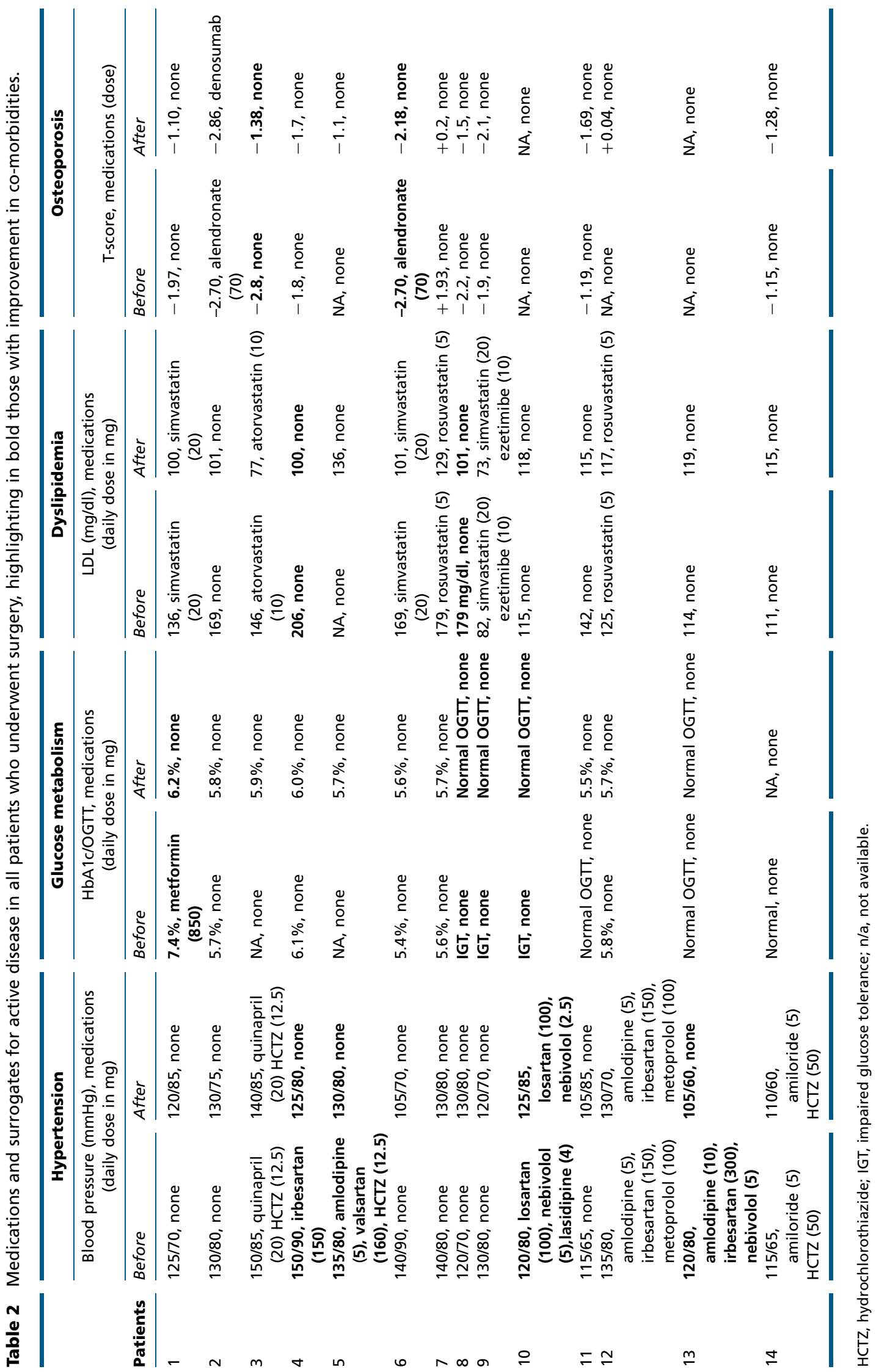


Table 3 Baseline biochemical characteristics in patients with $\mathrm{SH}$ and improvement in co-morbidities in this subgroup, before and after surgery (group A) or in first and last follow-up (group B).

\begin{tabular}{|c|c|c|c|}
\hline Characteristics & $\begin{array}{c}\text { Group A } \\
\text { (SH) }(n=12)\end{array}$ & $\begin{array}{c}\text { Group B } \\
(\mathrm{SH})(n=11)\end{array}$ & $\boldsymbol{P}$ \\
\hline Cortisol post-LDDST $(\mu \mathrm{g} / \mathrm{dl})$ & $5.7 \pm 3.0$ & $3.7 \pm 1.4$ & NS \\
\hline 24 h UFC ( $\mu g / 24 h)$ & $125.13 \pm 43.7$ & $103.8 \pm 63.5$ & NS \\
\hline $\operatorname{MSF}(\mu \mathrm{g} / \mathrm{dl})$ & $8.8 \pm 5.0$ & $6.9 \pm 2.5$ & NS \\
\hline ACTH $(p g / m l)$ & $7.1 \pm 5.2$ & $8.9 \pm 5.1$ & NS \\
\hline Improvement of hypertension & $4 / 6$ & $0 / 6$ & 0.01 \\
\hline Improvement of DM2 or IGT & $4 / 4$ & $0 / 2$ & 0.01 \\
\hline Improvement of dyslipidemia & $2 / 7$ & $0 / 3$ & NS \\
\hline Improvement of osteoporosis & $2 / 3$ & $0 / 4$ & 0.05 \\
\hline
\end{tabular}

$\mathrm{SH}$, subclinical hypercortisolism; LDDST, low dose dexamethasone suppression test; UFC, urinary free cortisol; MSF, midnight serum cortisol; DM2, type 2 diabetes; IGT, impaired glucose tolerance.

life-threatening adrenal crisis. Moreover, glucocorticoid replacement often exceeds the amount actually needed and cannot mimic the diurnal profile of endogenous cortisol, resulting in persisting exposure to subtle cortisol excess $(9,15)$.

These considerations led to a limited number of case reports or small studies to suggest that unilateral adrenalectomy might be a useful alternative. These studies, however, comprise of either case reports or a small number of patients, most have a short time of follow-up and, importantly, there is no control group. The population included in these studies comprises mainly of patients with overt Cushing's syndrome due to macronodular or micronodular adrenal hyperplasia $(11,16,17,18,19)$. Two studies included patients with subclinical hypercortisolism $(12,20)$. In all of these patients a significant improvement in the hormonal profile is reported. Although co-morbidities were not systematically studied, amelioration in diabetes and hypertension is reported, at least in a significant number of patients. In both studies the follow-up period was short and there was no control group.

Our study, although retrospective in nature, addresses these limitations and takes advantage of a control group with similar demographic, biochemical and clinical characteristics in which simple observation was chosen. Despite not being associated with typical signs and symptoms of Cushing's syndrome, it is now well appreciated that $\mathrm{SH}$ can lead to similar co-morbidities, such as hypertension, hyperglycemia, dyslipidemia and low bone density (4). Our study supports the link between the presence of cortisol excess and these morbidities, because they improved in a significant number of patients after the surgical correction of hypercortisolism, whereas they persisted in the conservatively managed group. Of note, a recent study reported increased cardiovascular events and mortality in patients with AI and lack of post-dexamethasone cortisol suppression below $1.8 \mu \mathrm{g} / \mathrm{dl}$. We previously showed that the prevalence of $\mathrm{SH}$ is higher among patients with bilateral vs unilateral AIs, although this was not associated with an increased prevalence of comorbidities (8). Another important strength of our study is the relatively long follow-up time. This is important because the improvement of co-morbidities was sustained for a long period of time and not confined only to the short post-operative period when even partial adrenal insufficiency may account for a reduction in glycemic levels or blood pressure. An important issue is whether some patients will eventually require completion adrenalectomy due to late recurrence of hypercortisolism. Our preliminary results show that the beneficial effects of unilateral adrenalectomy last for years, but a longer followup time will show whether they also last for lifetime.

Limitations of our study include its retrospective nature that cannot exclude possible selection biases toward treating those with more pronounced biochemical abnormalities. As a result, even though most subjects had at least one biochemical abnormality, the number of patients not fulfilling the criteria for $\mathrm{SH}$ in the follow-up group was higher, albeit not significantly different statistically. When comparing only subjects with $\mathrm{SH}$ in both groups, however, the observed improvements in co-morbidities remained significant. Also, we chose to remove the adrenal gland on the basis of larger size and not functional criteria (catheterization or iodocholesterol). In fact this criterion was used in most of the previous studies due to the limited experience and availability of these methods along with the so far successful results of this approach. In the study by Young et al. (12) lateralization of cortisol hypersecretion was at the side of the larger adrenal tumor in the majority of patients or was compatible with bilateral equal hypersecretion. Although our approach was rather empirical, it offered a clear benefit to the patients, possibly on the ground that larger adrenal nodules are more likely to represent the culprit lesion of $\mathrm{SH}$, or, in the case of bilateral cortisol production, removing the larger adrenal adenoma offers a significant debulking thus reducing cortisol excess. In case of equally sized lesions, our option was to opt for the less demanding surgery approach that is left adrenalectomy.

In conclusion, our results show that removal of the adrenal harboring the largest lesion offers significant improvement both to cortisol excess and metabolic consequences of hypercortisolism, without the debilitating 
effects of bilateral adrenalectomy. Bilateral adrenalectomy necessitates adrenal hormone replacement for life and is associated with higher morbidity compared to unilateral surgery; based on our results, the latter may be considered a useful management approach in patients with bilateral benign adrenal masses. These data, however, are preliminary, and a larger number of patients, as well as a longer follow-up of a prospective randomized study, is required before any solid conclusions can be established.

\section{Declaration of interest}

The authors declare that there is no conflict of interest that could be perceived as prejudicing the impartiality of the research reported.

\section{Funding}

This research did not receive any specific grant from any funding agency in the public, commercial or not-for-profit sector.

\section{References}

1 Kloos RT, Gross MD, Francis IR, Korobkin M \& Shapiro B. Incidentally discovered adrenal masses. Endocrine Reviews 199516 460-484. (doi:10. 1210/edrv-16-4-460)

2 Tsagarakis S, Vassiliadi D \& Thalassinos N. Endogenous subclinical hypercortisolism: diagnostic uncertainties and clinical implications. Journal of Endocrinological Investigation 200629 471-482. (doi:10.1007/ BF03344133)

3 Angeli A, Osella G, Ali A \& Terzolo M. Adrenal incidentaloma: an overview of clinical and epidemiological data from the national italian study group. Hormone Research 199747 279-283. (doi:10.1159/ 000185477)

4 Morelli V, Palmieri S, Salcuni AS, Eller-Vainicher C, Cairoli E, Zhukouskaya V, Scillitani A, Beck-Peccoz P \& Chiodini I. Bilateral and unilateral adrenal incidentalomas: biochemical and clinical characteristics. European Journal of Endocrinology 2013168 235-241. (doi:10.1530/EJE-12-0777)

5 Terzolo M, Pia A \& Reimondo G. Subclinical cushing's syndrome: definition and management. Clinical Endocrinology 201276 12-18. (doi:10.1111/j.1365-2265.2011.04253.x)

6 Vassiliadi DA \& Tsagarakis S. Endocrine incidentalomas - challenges imposed by incidentally discovered lesions. Nature Reviews. Endocrinology 20117 668-680. (doi:10.1038/nrendo.2011.92)

7 Chiodini I, Morelli V, Salcuni AS, Eller-Vainicher C, Torlontano M, Coletti F, Iorio L, Cuttitta A, Ambrosio A, Vicentini L et al. Beneficial metabolic effects of prompt surgical treatment in patients with an adrenal incidentaloma causing biochemical hypercortisolism. Journal of Clinical Endocrinology and Metabolism 201095 2736-2745. (doi:10.1210/ jc.2009-2387)

8 Vassiliadi DA, Ntali G, Vicha E \& Tsagarakis S. High prevalence of subclinical hypercortisolism in patients with bilateral adrenal incidentalomas: a challenge to management. Clinical Endocrinology 201174 438-444. (doi:10.1111/j.1365-2265.2010.03963.x)

9 Grossman A, Johannsson G, Quinkler M \& Zelissen P. Therapy of endocrine disease: perspectives on the management of adrenal insufficiency: clinical insights from across Europe. European Journal of Endocrinology 2013169 R165-R175. (doi:10.1530/ EJE-13-0450)

10 Ito T, Kurita Y, Shinbo H, Otsuka A, Furuse H, Mugiya S, Ushiyama T, Ozono S, Oki Y \& Suzuki K. Successful treatment for adrenocorticotropic hormone-independent macronodular adrenal hyperplasia with laparoscopic adrenalectomy: a case series. Journal of Medical Case Reports 20126 312. (doi:10.1186/1752-1947-6-312)

11 Ogura M, Kusaka I, Nagasaka S, Yatagai T, Shinozaki S, Itabashi N, Nakamura T, Yokoyama M, Ishikawa SE \& Ishibashi S. Unilateral adrenalectomy improves insulin resistance and diabetes mellitus in a patient with ACTH-independent macronodular adrenal hyperplasia. Endocrine Journal 200350 715-721. (doi:10.1507/endocrj.50.715)

12 Young WF Jr, du Plessis H, Thompson GB, Grant CS, Farley DR, Richards ML, Erickson D, Vella A, Stanson AW, Carney JA et al. The clinical conundrum of corticotropin-independent autonomous cortisol secretion in patients with bilateral adrenal masses. World Journal of Surgery 200832 856-862. (doi:10.1007/s00268-007-9332-8)

13 Vassiliadi DA, Ntali G, Stratigou T, Adali M \& Tsagarakis S. Aberrant cortisol responses to physiological stimuli in patients presenting with bilateral adrenal incidentalomas. Endocrine 201140 437-444. (doi:10.1007/s12020-011-9490-1)

14 Expert Committee on the D. Classification of Diabetes M. Report of the expert committee on the diagnosis and classification of diabetes mellitus. Diabetes Care 200326 (Suppl 1) S5-S20. (doi:10.2337/diacare. 26.2007.S5)

15 Plat L, Leproult R, L'Hermite-Baleriaux M, Fery F, Mockel J, Polonsky KS $\&$ Van Cauter E. Metabolic effects of short-term elevations of plasma cortisol are more pronounced in the evening than in the morning. Journal of Clinical Endocrinology and Metabolism 199984 3082-3092. (doi:10.1210/jcem.84.9.5978)

16 Iacobone M, Albiger N, Scaroni C, Mantero F, Fassina A, Viel G, Frego M \& Favia G. The role of unilateral adrenalectomy in ACTH-independent macronodular adrenal hyperplasia (aimah). World Journal of Surgery 200832 882-889. (doi:10.1007/s00268-007-9408-5)

17 Mazzuco TL, Chaffanjon P, Martinie M, Sturm N \& Chabre O. Adrenal cushing's syndrome due to bilateral macronodular adrenal hyperplasia: prediction of the efficacy of $\beta$-blockade therapy and interest of unilateral adrenalectomy. Endocrine Journal 200956 867-877. (doi:10.1507/endocrj.K08E-370)

18 Xu Y, Rui W, Qi Y, Zhang C, Zhao J, Wang X, Wu Y, Zhu Q, Shen Z, Ning $\mathrm{G}$ et al. The role of unilateral adrenalectomy in corticotropinindependent bilateral adrenocortical hyperplasias. World Journal of Surgery 201337 1626-1632. (doi:10.1007/s00268-013-2059-9)

19 Albiger NM, Ceccato F, Zilio M, Barbot M, Occhi G, Rizzati S, Fassina A, Mantero F, Boscaro M, Iacobone $\mathrm{M}$ et al. An analysis of different therapeutic options in patients with Cushing's syndrome due to bilateral macronodular adrenal hyperplasia: a single-centre experience. Clinical Endocrinology 201582 808-815. (doi:10.1111/cen.12763)

20 Papierska L, Cwikla J, Rabijewski M, Glinicki P, Otto M \& Kasperlik-Zaluska A. Bilateral adrenal incidentaloma with subclinical hypercortisolemia: indications for surgery. Polskie Archiwum Medycyny Wewnetrznej 2014124 387-394.

Received 7 June 2015

Revised version received 13 August 2015

Accepted 1 September 2015 